\title{
Price Trust Evaluation in E-Service Oriented Applications
}

\author{
Yan Wang \\ Department of Computing \\ Macquarie University \\ Sydney, NSW 2109 \\ Australia \\ yanwang@ics.mq.edu.au
}

\author{
Lei Li \\ Department of Computing \\ Macquarie University \\ Sydney, NSW 2109 \\ Australia \\ leili@ics.mq.edu.au
}

\author{
Ee-Peng Lim \\ Division of Information Systems \\ School of Computer Engineering \\ Nanyang Technological University \\ Singapore 639798 \\ aseplim@ntu.edu.sg
}

\begin{abstract}
Trust is a critical issue in e-commerce and e-service environments. Some applications (such as eBay) have introduced various trust management mechanisms to provide valuable information to buyers prior to placing orders and making payments. While trust has been actively studied in the research community, some malicious sellers and fraudulent transactions still widely exist. A typical attack is to entice buyers into making payments for buying items at very cheap and attractive prices and not to deliver the items or deliver the fake item after payment. This paper proposes a new trust management system that provides information to indicate to some extent the trust level of a seller at an offered price. In this paper, we describe our proposed approach for price trust evaluation and an approach for market price calculation. We also evaluated our approaches empirically.
\end{abstract}

\section{Introduction}

In some e-commerce or e-service applications, reputation-based trust management has been set up to provide trust information about sellers or service providers to buyers or service customers. This is important as any buyer or service customer would like to have transactions with reputable sellers or service providers so as to reduce the possibility to be deceived. This is particularly important when a customer has to select from unknown sellers or service providers.

In general, in a trust management mechanism enabled environment, buyers or service customers can provide feedback and ratings after transactions. The trust management system can calculate the trust value based on collected ratings to reflect the qualities of transactions in a certain period. The trust value can be provided to customers, by publishing it on the web or responding to their requests. In some studies (e.g. [9]), the temporal dimension is also taken into account. For instance, later transactions are given more weights as they are more recent and relevant to new transactions.

Peer-to-Peer networks (P2P) can be used for information-sharing systems ${ }^{1}$, where the trust issue is also actively studied. In a $\mathrm{P} 2 \mathrm{P}$ system, it is quite natural for a client peer to doubt if a serving peer can provide the complete version of the requested file prior to any download action, which may be quite time-consuming and network bandwidth-consuming. Different from some trust management systems in e-commerce environments, in the $\mathrm{P} 2 \mathrm{P}$ trust system, a requesting peer needs to enquire the trust data of a serving peer (target peer) from other peers which may have transacted with the serving peer in the past $[1,3,8]$. The computation of the trust status of the serving peer from the collected trust ratings is then performed by the requesting peer, not a central management server. This is because of the decentralized architecture of P2P systems.

In existing trust management studies, the final trust value is computed to reflect the general or global trust status of every service provider. However, it is good but not directly relevant to the forthcoming transaction. A typical attack is that a malicious seller can cheat customers by offering a much cheaper price to attract mass orders from buyers. After the payment, a buyer may receive nothing or receive a product which is not the genuine version. Thus a good trust management system is expected to provide more (precise) trust information that indicates the trust level with respect to an offered price. It is also to some extent an indication of the risk level of the new transaction. In this paper, we propose the notion of price trust and its method of evaluation. In addition, we propose an algorithm for calculating market price. The two approaches correlate to each other and lead to an iterative process for computation.

This paper is organized as follows. In Section 2, we review the trust management in applications and some exist-

${ }^{1}$ www.gnutella.com 
ing studies. Section 3 discusses the metrics of price trust evaluation and presents our proposed approaches. Some empirical studies are presented in Section 4 for further illustrating the properties of our model. Finally Section 5 concludes our work.

\section{Background}

\subsection{Related Work}

eBay $^{2}$ is a typical Customer-to-Customer (C2C) web site. Its trust management mechanism is one of the earliest deployed systems in e-commerce applications. At eBay, after each transaction, the buyer can give feedback according to the service quality of the seller. The feedback (in the form of rating) is centrally stored by eBay. The feedback can be "positive", "neutral" or "negative". eBay calculates the feedback score $S=P-N$, where $P$ is the number of positive feedback responses and $N$ is the number of negative feedback responses. $S$ value is displayed on the web page. $R=\frac{P-N}{P+N}$ (e.g., $R=99.1 \%$ ) is called positive feedback rate, based on which the seller can be awarded as a "Power Seller" if $R \geq 98 \%$ ( $98 \%$ is the threshold).

eBay also improves its trust service and provides rating data in 12 months listed in a table, which is divided by recent 1 month, 6 months and 12 months. Thus, eBay provides some simple mechanisms of trust management and trust calculation and leaves some raw data to buyers for selfjudgement. There is however much room for improvement - such as, recent ratings are more important in the trust evaluation [9]; it does not divide sellers into multiple classes of reputation ranks either (e.g. a 5-star seller, or a 4-star seller) as in [4].

P2P network technology has been widely used in information-sharing systems, where a peer (serving peer) can share some information (e.g. music files or movie files) with other peers (client peers) without any central management authority. In this environment, the issue that a file being provided is complete or not is the concern of a client peer before any download action. EigenTrust [1] collected the local trust values of all peers to calculate the global trust value of a given peer. In [3], Marti and Garcia-Molina proposed a voting reputation system targeting at e-commerce applications that collects responses from other peers on a given peer. The final reputation value is calculated by combining the values returned by responding peers and the requesting peer's experience with the given peer.

In the literature, trust issue also received much attention in service-oriented computing research. In [2], Lin et al proposed a method of reputation-based trust evaluation in service-oriented environments based on a proposed ar-

\footnotetext{
${ }^{2}$ www.ebay.com
}

chitecture consisting of distributed trust management brokers. In [5], Vu et al proposed a model to evaluate and rank the trust and reputation of QoS-based services. In [7], an event-driven and rule-based trust management for serviceoriented application is proposed, where a formula based approach is adopted for incremental trust computation. The approach is adaptable to applications by incorporating rule management. The computed result can be taken as a global trust value reflecting the accumulated trust level, which is not particularly relevant to a forthcoming transaction.

\subsection{Price Trust}

In existing studies, in general, the proposed approaches are used to calculate the global or general trust value, which is based on feedback collected from customers. Such a trust value is assumed to be able to indicate the success possibility or trustworthiness degree of the new transaction. However, as we have pointed in Section 1, a good trust management should indicate the trust level with respect to an offered price, which may not be based on collected trust ratings. This is a realistic task particularly for a centralized trust management system (say eBay) as it relies on the mass of sellers registered at the web site and it is not a difficulty to collect the prices from sellers for the same product or service, based on which the price trust can be calculated. In contrast, in most existing studies, the price or transaction amount is not taken into account in trust evaluation except the work in [6], which aims to prevent the attack to deceive buyers by selling expensive products after obtaining good reputation by selling cheap products.

\section{Price Trust Evaluation}

In this section, we will present the data structure for trust evaluation, and some trust evaluation metrics, based on which we will propose the price trust evaluation method.

\subsection{Trust Data Structure}

In order to calculate the price trust, we assume the following trust data structure.

$$
T R=<S, C, R_{C \rightarrow S}^{(t)}, P, p, t>
$$

where

1. $T R$ is the transaction occurred at time $t$ between seller or service provider $S$ and buyer or service customer $C$;

2. $P=p d(T R)$ is the product purchased in the transaction;

3. $p=p r(T R)$ is the price for the product or service purchased in the transaction; 
4. $R_{C \rightarrow S}^{(t)}=\operatorname{rating}(T R) \in[0,1]$ is the rating given by $C$;

5. In addition, we use $\operatorname{tran}\left(R_{C \rightarrow S}\right)$ to denote transaction $T R$.

\subsection{Price Trust Evaluation Metrics}

In general, a buyer is concerned about whether the offered price is a normal one in the market. If it is too high or too low, it indicates high transaction risk level and thus low transaction trust. In particular, some malicious sellers aim to attract buyers by offering quite low price then cheat them. The Price Trust (PT) is expected to identify this type of cases and leave risk indication to potential buyers.

Price trust results from the comparison of the offered price and the market price. High price trust value results from low price difference. High price difference leads to low price trust value. This trust value is useful to prevent the fraudulent transaction by offering low and attractive price.

In general, the price trust can be computed from the distance of offered price and the market price. Namely, if the offered price is much lower than the market price, it indicates a low price trust and a high transaction risk. Meanwhile, if the offered price is much higher than the market price, it also certainly indicates a high transaction risk and low price trust. Thus, a trustworthy offered price should be close to the market price with the distance in a certain scope.

Let $\delta_{p}=\frac{o p-m p}{m p}$ denote the price distance in percentage, where $o p$ is the offered price and $m p$ is the market price. Some principles for calculating price trust are discussed as follows.

Principle 1: The price trust value can be in the range of $[0,1]$, where 0 represents the lowest trust level and 1 represents the highest trust level.

Principle 2: If the offered price $o p$ is in the normal range in $\left[p_{-}, p_{+}\right]\left(0<p_{-}<p_{+}\right), P T$ is considered as 1.0. Here $\delta_{p}$ is redefined as follows.

$$
\delta_{p}=\left\{\begin{array}{cl}
\frac{o p-p_{+}}{p_{+}} & \text {if } o p>p_{+} \\
0 & \text { if } p_{-} \leq o p \leq p_{+} \\
\frac{o p-p_{-}}{p_{-}} & \text {if } o p<p_{-}
\end{array}\right.
$$

In this case, one option is to take $p_{r}$, the price recommended by the manufacturer, as the upper bound $p_{+}$. As $p_{r}$ is usually more than $m p$ for a certain extent, an offered price which is more than $p_{r}$ is taken as less trustworthy. Meanwhile, this option may be productdependant as for some products (e.g. digital cameras), as $p_{r}$ is usually much higher than $m p$ by $15-25 \%$.
There is another option to take $p_{e}$, the price in the estore or e-shop of the manufacturer, as $p_{+}$. If $m p$ is higher than $p_{e}$, buyers will choose the e-store of the manufacturer, since it is more trustworthy and cheaper. So, in practice, $p_{e}$ is usually higher than $m p$.

In contrast, $p_{-}$, the lower bound of normal price, is hard to be determined. One option is to define an extent within which the price is considered as normal. For example, if the offered price is up to $e=10 \%$ less than the market price, it is normal. Meanwhile, the determination of $p_{-}$is also product-dependant.

Principle 3: $P T \in[0,1)$ is a function of $\delta_{p}$, and its value is in reverse proportion to $\left|\delta_{p}\right|$, if $\delta_{p} \neq 0$.

Principle 4: $P T$ is a continuous or approximately continuous function of $\delta_{p}$.

If there is a discontinuous point $\delta_{0}$, the $P T$ for $\delta_{0}$ is much different from the one for $\delta_{0}+\epsilon(\epsilon$ is an arbitrary small positive value in $(0,1))$. However, it is a controversy since $P T$ has no such jump point.

Principle 5: If $\left|\delta_{p_{1}}\right|=\left|\delta_{p_{2}}\right|, \delta_{p_{1}}<0$ and $\delta_{p_{2}}>0, P T_{1}$ may be different from $P T_{2}$, where $P T_{1}$ and $P T_{2}$ are the corresponding $P T$ for $\delta_{p_{1}}$ and $\delta_{p_{2}}$ respectively.

This principle can be further divided into two cases.

Principle 5.1: If both $\left|\delta_{p_{1}}\right|$ and $\left|\delta_{p_{2}}\right|$ are close to 0 , $P T_{1} \geq P T_{2}$.

This principle aims to leave some space to seller who may try to attract customers by offering low prices. Namely, if $o p$ is less than but very close to $p_{-}, P T$ is close to 1.0 .

Principle 5.2: If both $\left|\delta_{p_{1}}\right|$ and $\left|\delta_{p_{2}}\right|$ are not close to $0, P T_{1}<P T_{2}$.

This principle aims to identify a case of cheating by offering very low price. For example, normally it is quite difficult to sell a product $50 \%$ lower than $m p$. In this case, it is more likely to be a cheating case that the buyer will not receive the product ordered. But it is a bit normal to offer a price $50 \%$ higher than $m p$. This is more likely for the seller to earn more money rather than cheat the buyer.

\subsection{Price Trust Evaluation Method}

In this section, we will design the evaluation method for price trust.

Let $P T_{S(P)}$ denote the price trust of seller $S$ with price op of product $P$. According to Principles 1 and 2, given $p_{-}, p_{+}$and $o p \in\left[p_{-}, p_{+}\right], P T_{S(P)}=1$. 
Now let's consider the case of $\delta_{p}=\frac{o p-p_{-}}{p_{-}}<0$, where op $<p_{-}$. According to Principle 3, a larger $\left|\delta_{p}\right|$ results in a smaller $P T$, which approaches 0 . To simulate this property, we adopt the Hyperbolic Tangent function and transform it into our formula as follows.

$$
P T_{S(P)}=\frac{e^{\nu \cdot \eta}-e^{-\nu \cdot \eta}}{2\left(e^{\nu \cdot \eta}+e^{-\nu \cdot \eta}\right)}+0.5, \quad \text { if } \delta_{p} \in[-1,0),
$$

where $\eta=2 \cdot \delta_{p}+1$, and $\nu \geq 1$ is the argument for controlling the function curve. As the Hyperbolic Tangent function

$$
f(x)=\frac{e^{x}-e^{-x}}{e^{x}+e^{-x}}
$$

is in the range of $[0,1)$, it is easy to know that $P T_{S(P)} \in$ $(0,1)$.

For the case of $\delta_{p}=\frac{o p-p_{+}}{p_{+}}>0$, it is similar but slightly different from the case of $\delta_{p}<0$. Firstly, in the case of $\delta_{p}<0, \delta_{p}$ is in the range of $[-1,0)$. However, if $\delta_{p}>0$, it is possible that $\delta>1$. Secondly, the change trend of $P T$ is slightly different from the case of $\delta_{p}<0$. In the design of formula (3), when $o p<p_{-}$but $o p$ is very close to $p_{-}$, PT is close to 1 . However, if $o p$ is away from $p_{-}$by a certain extent, $P T$ drops quickly. In contract, when $o p>p_{+}$, $P T$ starts to drop dramatically from 1 . In addition, as it is possible for $\delta_{p}$ to be more than $1, P T$ approaches to 0 more slowly than that in the case of $\delta_{p}<0$. Namely, according to Principle 5.2, given $\left|\delta_{p_{1}}\right|=\left|\delta_{p_{2}}\right|, \delta_{p_{1}}<0$ and $\delta_{p_{2}}>0$, both $\delta_{p_{1}}$ and $\delta_{p_{1}}$ are not very close to $0, P T_{1}<P T_{2}$. Based on the above analysis, we design a formula as follows for the evaluation of PT by adopting the transformation of the Hyperbolic Secant function.

$$
P T_{S(P)}=\frac{2}{e^{\delta_{p} \cdot \gamma}+e^{-\delta_{p} \cdot \gamma}}, \quad \text { if } \delta_{p} \geq 0,
$$

where $\gamma \geq 1$ is the argument for controlling the function's curve.

Definition 1: Let $o p$ and $m p$ denote the offered price of seller $S$ and the market price of product $P$ respectively, and

$$
\delta_{p}=\left\{\begin{array}{cl}
\frac{o p-p_{+}}{p_{+}} & \text {if } o p>p_{+} \\
0 & \text { if } p_{-} \leq o p \leq p_{+} . \\
\frac{o p-p_{-}}{p_{-}} & \text {if } o p<p_{-}
\end{array}\right.
$$

The price trust $P T$ is calculated as

$$
P T_{S(P)}= \begin{cases}1.0 & \text { if } o p \in\left[p_{-}, p_{+}\right] \\ \frac{2}{e^{\delta} p^{\cdot \gamma}+e^{-\delta_{p} \cdot \gamma}} & \text { if } \delta_{p} \geq 0 \\ \frac{e^{\nu \cdot \eta}-e^{-\nu \cdot \eta}}{2\left(e^{\nu \cdot \eta}+e^{-\nu \cdot \eta}\right)}+0.5 & \text { if } \delta_{p} \in[-1,0)\end{cases}
$$

where $\eta=2 \cdot \delta_{p}+1, \gamma \geq 1$ and $\nu \geq 1$ are arguments for controlling the function curve. The setting of $\gamma$ and $\nu$ will be studied in section 4.1 .

According to the above definition, as the PT function is transformed from the Hyperbolic Secant (in $[0,1)$ ) and Hyperbolic Tangent (in $(-1,1)$ ) functions respectively, $P T$ is in the range of $(0,1]$. Other properties will be studied in Section 4.1.

\subsection{Market Price}

In a centralized architecture (like eBay), as all sellers or service providers are providing services and making transactions on the same web site, it is easy to collect all prices of a certain product or service. In a simple case, the market price of product $P$ can be calculated as the mean of all prices offered to customers. Namely,

Definition 2: Let $o p_{i}$ denote an offered price of product $P$, the market price of $P$ in a naive strategy can be calculated as follows:

$$
m p_{P}=\frac{1}{h} \sum_{S_{i} \text { is a seller of } P} o p_{i},
$$

where $h$ is the number of sellers selling $P$.

In Eq. (7), there is an assumption that all offered prices are trustworthy. In practice, Eq. (7) may contain some noise if all offered prices are taken into account. Thus, in the computation of the market price, the trustworthiness of an offered price should be taken in account.

With $m p_{P}$, the PT values can be calculated for each price, after which the new market price can be computed after filtering out some less trustworthy prices.

Definition 3: Let $o p_{i}$ denote an offered price of product $P$, the trustworthy market price of $P$ can be calculated as follows:

$$
m p_{P}^{\prime}=\frac{1}{h^{\prime}} \sum_{P T\left(o p_{i}\right) \geq \rho} o p_{i}
$$

where $h^{\prime}$ is the number of sellers whose PT is no worse than a threshold $\rho$, i.e. $P T\left(o p_{i}\right) \geq \rho$.

However, the above method does not take any price trust into account when calculating the market price. Instead, $P T$ is used for filtering out low trust prices. Alternatively, the $P T$ of each trustworthy price can be taken as a weight when calculating the market price. Thus, we propose a new market price evaluation method as follows.

Definition 4: Let $o p_{i}$ denote an offered price of product $P$, the trustworthy market price of $P$ can be calculated as follows:

$$
m p_{P}^{\prime \prime}=\frac{\sum_{P T\left(o p_{i}\right) \geq \rho} P T\left(o p_{i}\right) \cdot o p_{i}}{\sum_{P T\left(o p_{i}\right) \geq \rho} P T\left(o p_{i}\right)} .
$$

Based on the above discussion, the calculation of PT is an iterative process as with the new market price, the PT value for each offered price will be re-calculated. The new PT values may yield some low trustworthiness prices to be filtered out and thus the new market price. This iterative process can be repeated until each value becomes stable.

The iterative algorithm is described as follows:

Algorithm 1 Compute market price $m p$.

Input: the offered price $o p_{i}$ of each seller,

the e-store price $p_{e}$,

the threshold $\lambda$, and

an arbitrary small positive number $\epsilon$ (such as 0.01 ).

Output: $m p$ 


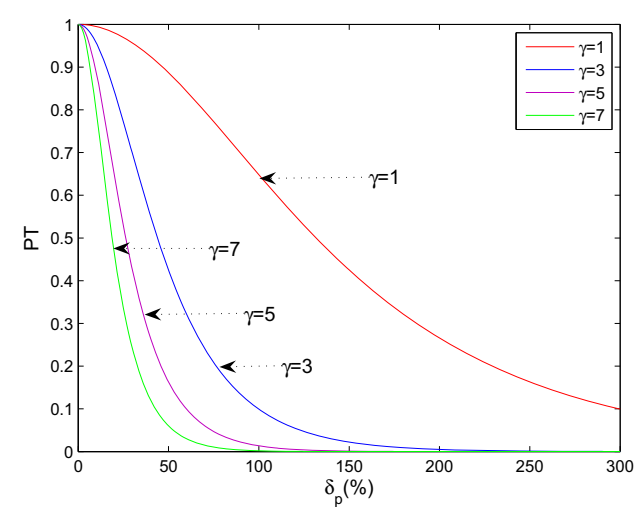

Figure 1. PT when $\delta_{p} \geq 0$

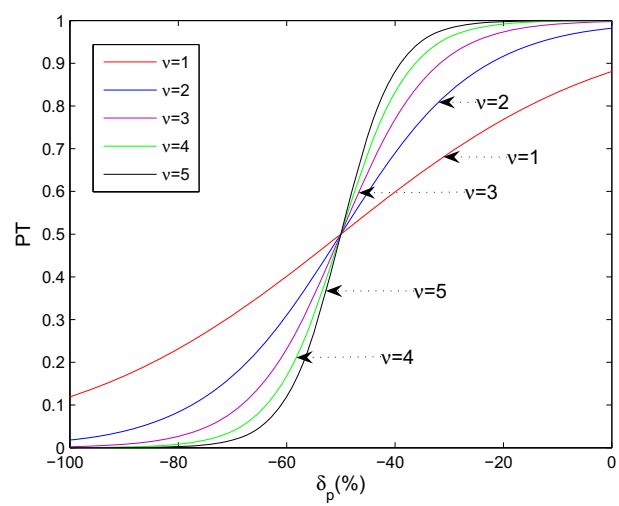

Figure 2. PT when $\delta_{p} \leq 0$

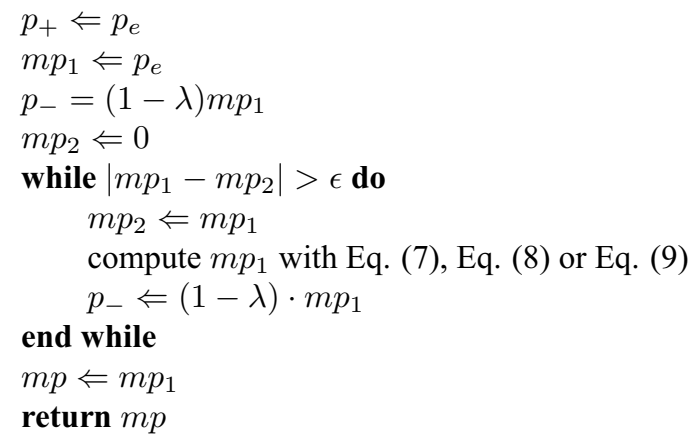

\section{Empirical Study}

In this section, we will firstly study the properties of our proposed models, after which we will conduct some case studies and apply our models to some products being sold at eBay and Taobao.com.

\subsection{Study 1}

In this section, we study the properties of formula (6) for calculating the Price Trust. We set $\gamma=1,3,5$, or 7 ,

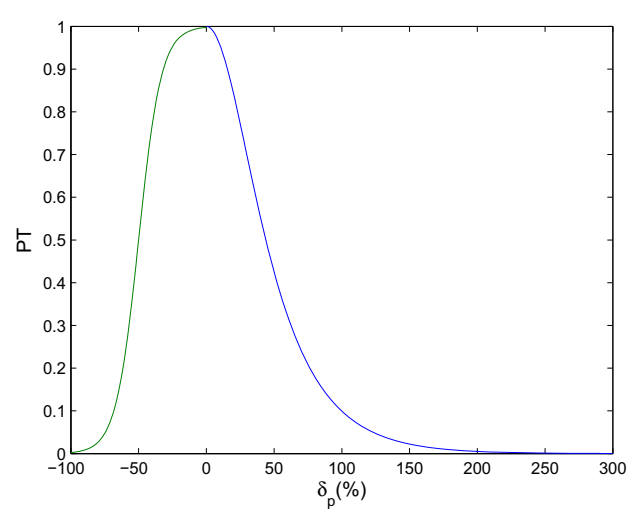

Figure 3. PT when $\gamma=3, \nu=3$

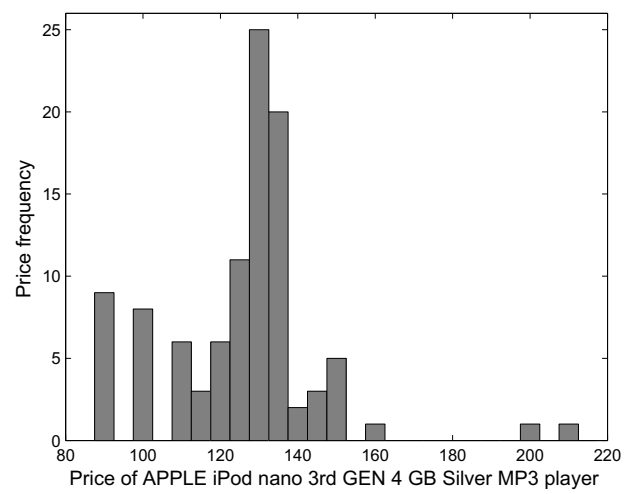

Figure 4. Price frequency in Study 2 (from eBay)

$\nu=1,2,3,4$, or 5 and observe the function curve changes.

In Fig. 1, when $\delta_{p}=50 \%, P T=0.89,0.44,0.17$, or 0.064 if $\gamma=1,3,5$, or 7 respectively. When $\delta_{p}=100 \%$, $P T=0.65,0.1,0.014$, or 0.002 if $\gamma=1,3,5$, or 7 respectively. Relatively, the result is more reasonable when $\gamma \in[3,5]$.

Similarly, the function curve changes can be observed in Fig. 2 for the case of $\delta_{p} \leq 0$ when setting $\nu=1,2,3,4$ or 5. A larger $\nu$ value results in steeper curve. We can observe that when $\nu \geq 3$, the trust value is close to 1.0 if $\delta_{p}=-10 \%$ or $-20 \%$. But PT starts to drop when $\delta_{p}$ is less than $-30 \%$. This is reasonable to reflect Principle 5. Meanwhile, from Fig. 2, we can see that $P T$ function satisfies Principle 4 when $\nu \geq 3$.

When selecting $\gamma=3$ and $\nu=3$, PT formula (6) is plotted in Fig. 3, which is used for PT evaluation in the following studies.

\subsection{Study 2}

In this study, we apply our models to a product offered at eBay: APPLE iPod nano 3rd GEN 4 GB Silver MP3 player. 
The corresponding histogram of prices and price frequency are depicted in Fig. 4. This product is also offered in the e-store of the manufacturer ${ }^{3}$, where its price is $p_{e}=149.00$ USD.

In this case, we collect 101 prices, and the range of all prices is from 91 USD to 210 USD. From Fig. 4, we can observe that this example contains minor low price noise, and most prices are not far away from $p_{e}$. For example, there are 25 prices offered in the range of 130 USD to 135 USD.

In this study, we set the threshold $\lambda=0.05$ in Algorithm 1 , and $\rho=0.8$ or 0.9 in Eqs (8) and (9). The computed $m p_{P}, m p_{P}^{\prime}$ and $m p_{P}^{\prime \prime}$ are listed in Table 1. The corresponding PT values are calculated and plotted in Fig. 5 and Fig. 6.

We notice from Fig. 5 and Fig. 6 that the three different price trust values $P T_{o p}, P T_{o p}^{\prime}$ and $P T_{o p}^{\prime \prime}$ have minor difference because of minor low trust prices. Therefore, due to the same reason, $m p_{P}, m p_{P}^{\prime}, m p_{P}^{\prime \prime}$ are also close to each other (see Table 1).

\begin{tabular}{|c|c|c|c|c|c|}
\hline$\rho$ & $\lambda$ & $m p_{P}$ & $m p_{P}^{\prime}$ & $m p_{P}^{\prime \prime}$ & Figure \\
\hline 0.8 & 0.05 & 125.53 & 123.92 & 124.08 & Fig. 5 \\
\hline 0.9 & 0.05 & 125.53 & 123.98 & 124.11 & Fig. 6 \\
\hline
\end{tabular}

Table 1. Study 2

\begin{tabular}{|c|c|c|c|c|c|}
\hline$\rho$ & $\lambda$ & $m p_{P}$ & $m p_{P}^{\prime}$ & $m p_{P}^{\prime \prime}$ & Figure \\
\hline 0.8 & 0.05 & 599.58 & 621.90 & 624.57 & Fig. 8 \\
\hline 0.9 & 0.05 & 599.58 & 648.08 & 648.59 & Fig. 9 \\
\hline
\end{tabular}

Table 2. Study 3

\subsection{Study 3}

In this study, we present another example: Nokia N95 $8 \mathrm{~GB}$ cell phone, which is offered at eBay. The corresponding histogram is plotted in Fig. 7, and the e-store price of the manufacturer is $p_{e}=779 \mathrm{USD}^{4}$.

In this case, we collect 40 prices. The range of all prices is from 299 USD to 729 USD. From Fig. 7, we can observe that this example contains some low price noise and all prices are less than $p_{+}=p_{e}$.

We set the threshold $\lambda=0.05$, and $\rho=0.8$ or 0.9 . Based on Algorithm 1, we have calculated $m p_{P}, m p_{P}^{\prime}$, and $m p_{P}^{\prime \prime}$ which are listed in Table 2. The corresponding PT values are computed and plotted in Fig. 8 and Fig. 9.

From Fig. 8, we can observe that, for the same offered price $o p<p_{-}, P T_{o p}>P T_{o p}^{\prime}>P T_{o p}^{\prime \prime} . P T_{o p}^{\prime \prime}$ is the most

\footnotetext{
${ }^{3} \mathrm{http} / / /$ store.apple.com/1-800-MY-APPLE/WebObjects/AppleStore.wo a/wa/RSLID?nnmm=browse \&mco=3587D037\&node=home/shop_ipod/fa mily/ipod_nano

${ }^{4}$ http://www.nokiausa.com/link?cid=PLAIN_TEXT_358159
}

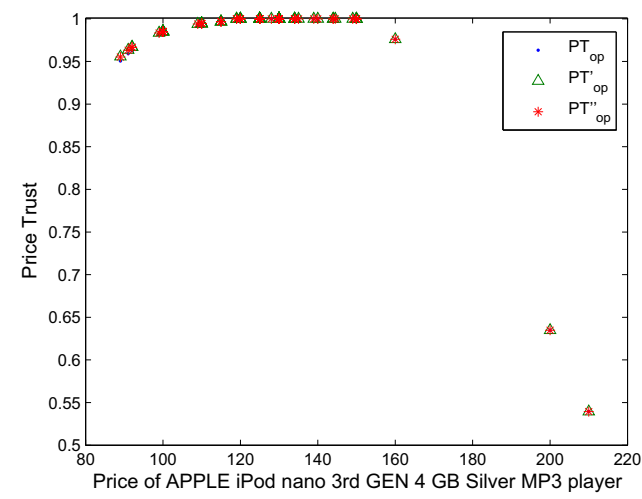

Figure 5. Price trust in Study 2 ( $\rho=0.8, \lambda=$ $0.05)$

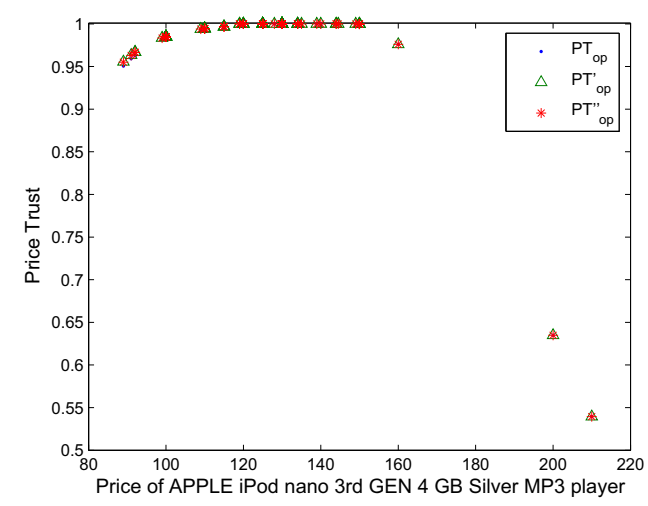

Figure 6. Price trust in Study 2 ( $\rho=0.9, \lambda=$ 0.05 )

desirable, as it indicates the most rigorous result. Correspondingly, $m p_{P}<m p_{P}^{\prime}<m p_{P}^{\prime \prime}$ (see Table 2).

We notice from Fig. 9 that, given an $o p$, the two price trust values $P T_{o p}^{\prime}$ and $P T_{o p}^{\prime \prime}$ are almost the same, because the threshold $\rho=0.9$ is high. Meanwhile, they are larger than $P T_{o p}$, as there are some low price noise in the computation of $P T_{o p}$ (see Table 2).

\subsection{Study 4}

In the end, we present a study on a product offered at Taobao.com (www.taobao.com): Sandisk Extreme III 4G Compact Flash card. Taobao.com is the most popular C2C web site in China. The corresponding histogram is illustrated in Fig. 10 and $p_{e}=485 \mathrm{CNY}(69.99 \mathrm{USD})^{5}$.

In this case, 26 prices are collected. The range of all prices is from $105 \mathrm{CNY}$ to $510 \mathrm{CNY}$. As illustrated in Fig.

\footnotetext{
${ }^{5} \mathrm{http} / / /$ shop.sandisk.comstoresdiskusen_USDisplayProductDetailsPage productID. 103443900
} 


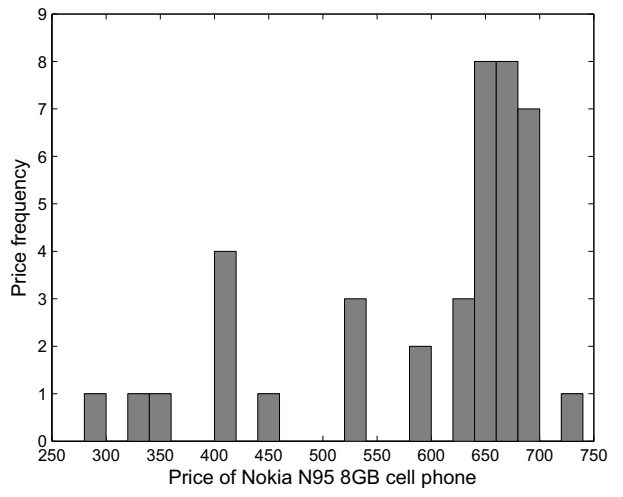

Figure 7. Price frequency in Study 3 (from eBay)

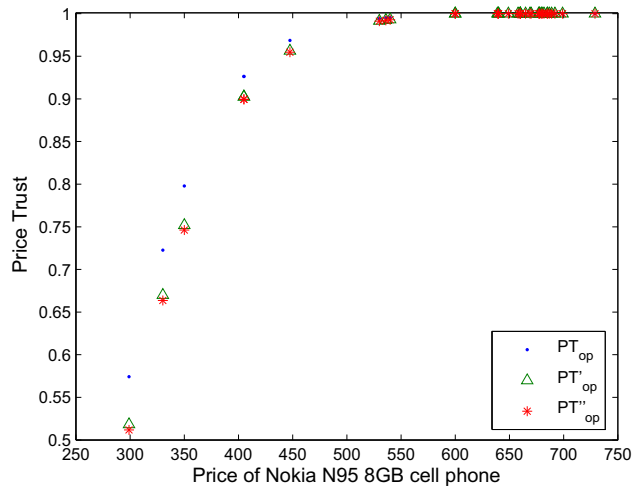

Figure 8. Price trust in Study $3(\rho=0.8, \lambda=$ $0.05)$

10 , this example contains much low price noise and minor high price noise.

In this study, we set the threshold $\lambda=0.05$, and $\rho=0.9$ or 0.5 . The computed $m p_{P}, m p_{P}^{\prime}$, and $m p_{P}^{\prime \prime}$ are listed in Table 3. The corresponding PT values are calculated and plotted in Fig. 11 and Fig. 12.

\begin{tabular}{|c|c|c|c|c|c|}
\hline$\rho$ & $\lambda$ & $m p_{P}$ & $m p_{P}^{\prime}$ & $m p_{P}^{\prime \prime}$ & Figure \\
\hline 0.9 & 0.05 & 287.96 & 344.35 & 344.32 & Fig. 11 \\
\hline 0.5 & 0.05 & 287.96 & 329.45 & 333.63 & Fig. 12 \\
\hline
\end{tabular}

Table 3. Study 4

We notice from Fig. 11 that, for the same offered price $o p$, the two price trust values $P T_{o p}^{\prime}$ and $P T_{o p}^{\prime \prime}$ are almost the same, because the threshold $\rho=0.9$ is high. Meanwhile, given op, $P T_{o p}^{\prime}$ and $P T_{o p}^{\prime \prime}$ are smaller than $P T_{o p}$, as there are much low trust noise. Therefore, $m p_{P}^{\prime}$ and $m p_{P}^{\prime \prime}$ are

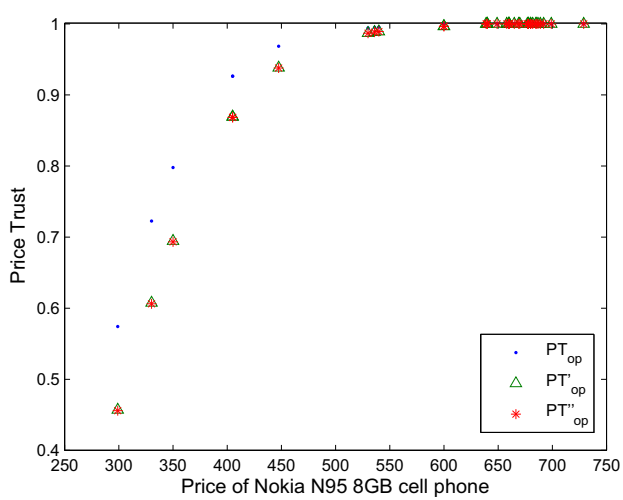

Figure 9. Price trust in Study 3 ( $\rho=0.9, \lambda=$ $0.05)$

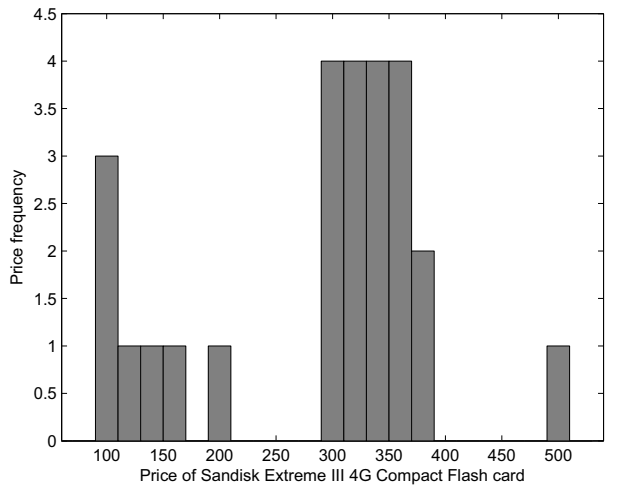

Figure 10. Price frequency in Study 4 (from Taobao)

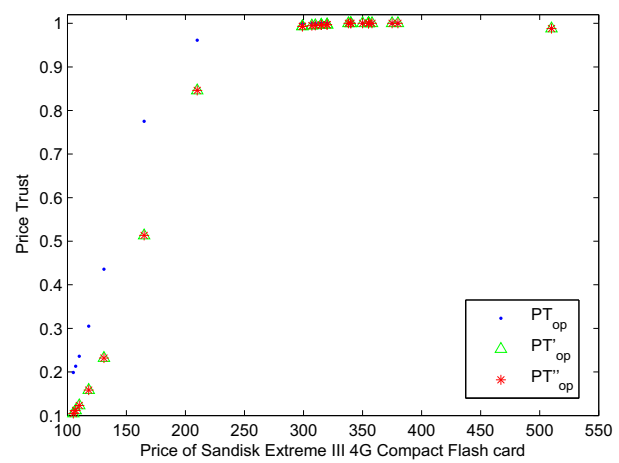

Figure 11. Price trust in Study $4(\rho=0.9, \lambda=$ 0.05 ) 


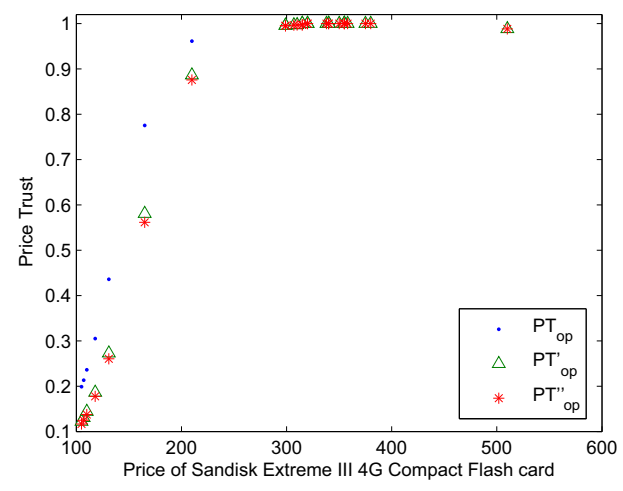

Figure 12. Price trust in Study $4(\rho=0.5, \lambda=$ $0.05)$

almost the same, which are more than $m p_{P}$ (see Table 3).

In contrast, when $\rho=0.5$, from Fig. 12, we can observe that, for the same offered price op $<p_{-}, P T_{o p}>$ $P T_{o p}^{\prime}>P T_{o p}^{\prime \prime}$. Similar to Study 3, $P T_{o p}^{\prime \prime}$ is the most desirable, as it indicates the most rigorous result. Correspondingly, $m p_{P}<m p_{P}^{\prime}<m p_{P}^{\prime \prime}$ (see Table 3 ).

In addition, when $\lambda=0.05$ and $\rho=0.9$, we notice that $P T_{107 \mathrm{CNY}}^{\prime \prime}=0.11$. It indicates that the corresponding price (i.e. $o p=107 \mathrm{CNY}$ ) has a low trust level and the product may have some quality problems. This is proved true as it is a faked card according the buyer's feedback ${ }^{6}$. Similarly, the product is proved to be a faked card for the case of $P T_{110 \mathrm{CNY}}^{\prime \prime}=0.12^{7}$ and the case of $P T_{130.9 \mathrm{CNY}}^{\prime \prime}=$ $0.23^{8}$.

In both study 3 and study $4, m p_{P}$ is much different from $m p_{P}^{\prime}$ and $m p_{P}^{\prime \prime}$ (in case of $\rho=0.8$ or 0.9 ). This is because of the introduction of PT, which helps filter out low trustworthiness prices and results in more trustworthy market price.

\section{Conclusions}

In this paper, we present the new concept of price trust and an approach for price trust evaluation. The trust result is particularly bound to a forthcoming transaction and can to some extent indicate the trust level or risk level of an offered price and the new transaction. In addition, we also present an iterative algorithm to market price calculation, which can

\footnotetext{
${ }^{6} \mathrm{http} / / /$ my.taobao.commytaobaoraterate.htm?user_id=22ea2d7b1f823b 5 fc96fdaabe3c92067\&time_line=\&buyer_or_seller=0\&good_neutral_or_ba $\mathrm{d}=0$ \&detailed $=\&$ received_or_posted $=0 \#$

${ }^{7} \mathrm{http}: / /$ my.taobao.commytaobaoraterate.htm?user_id $=475 \mathrm{f} 5674 \mathrm{dcc} 8 \mathrm{cdc}$ 7930648 bfbd31 f341\&detailed=1\&good_neutral_or_bad=-1\&time_line=$365 \&$ received_or_posted $=0 \&$ buyer_or_seller $=0 \#$

${ }^{8} \mathrm{http}: / /$ my.taobao.commytaobaoraterate.htm?user_id=f00baa3cbf05c69 fe34158a5f4e1909b\&time_line=\&buyer_or_seller=0\&good_neutral_or_bad $=0 \&$ detailed $=\&$ received_or_posted $=0 \#$
}

contribute to the calculation of the price trust. The computed price trust and market price are especially important and valuable to a buyer or a service customer prior to the new transaction.

For future work, the determination of $p_{-}$and $p_{+}$remains an open problem as it varies from product to product.

\section{References}

[1] S. D. Kamvar, M. T. Schlosser, and H. Garcia-Molina. The eigentrust algorithm for reputation management in P2P networks. In Proceedings of the 12th International WWW Conference, Budapest, Hungary, May 2003.

[2] K.-J. Lin, H. Lu, T. Yu, and C. en Tai. A reputation and trust management broker framework for web applications. In Proceedings of The 2005 IEEE International Conference on e-Technology, e-Commerce and eService (EEE'05), pages 262-269, March 2005.

[3] S. Marti and H. Garcia-Molina. Limited reputation sharing in $\mathrm{P} 2 \mathrm{P}$ systems. In Proceedings of ACMEC'04, pages 91-101, New York, USA, May 2004.

[4] S. Song, K. Hwang, R. Zhou, and Y.-K. Kwok. Trusted $\mathrm{p} 2 \mathrm{p}$ transactions with fuzzy reputation aggregation. IEEE Internet Computing, 9(6):24-34, 2005.

[5] L.-H. Vu, M. Hauswirth, and K. Aberer. Qos-based service selection and ranking with trust and reputation management. In Proceedings of 13th International Conference on Cooperative Information Systems (CoopIS 2005), Oct 31-Nov 42005.

[6] Y. Wang and F. Lin. Trust and risk evaluation of transactions with different amounts in peer-to-peer ecommerce environments. In Proceedings of The IEEE International Conference on e-Business Engineering (ICEBE 2006), pages 102-109, Shanghai, China, October 2006.

[7] Y. Wang, K.-J. Lin, D. S. Wong, and V. Varadharajan. The design of a rule-based and event-driven trust management framework. In The IEEE International Conference on e-Business Engineering (ICEBE 2007), pages 97-104, Hong Kong, October 2007.

[8] L. Xiong and L. Liu. PeerTrust: Supporting reputationbased trust for peer-to-peer electronic communities. IEEE Trans. on Knowlege and Data Engineering, 16(7):843-857, 2004.

[9] G. Zacharia and P. Maes. Trust management through reputation mechanisms. Applied Artificial Intelligence Journal, 9:881-908, 2000. 